\title{
An Erlang Multirate Loss Model Supporting Elastic Traffic under the Threshold Policy
}

\author{
Ioannis D. Moscholios ${ }^{1}$, Michael D. Logothetis ${ }^{2}$, Anthony C. Boucouvalas ${ }^{1}$ and Vassilios G. Vassilakis ${ }^{3}$ \\ 1. Dept. of Informatics \& Telecommunications, University of Peloponnese, 22100 Tripolis, Greece. \\ Email: \{idm, acb $\} @$ uop.gr \\ 2. WCL, Dept. of Electrical and Computer Engineering, University of Patras, 26504 Patras, Greece. \\ Email: mlogo@upatras.gr \\ 3. Dept. of Electronic Engineering, University of Surrey, GU2 7XH Guildford, U.K. \\ Email: v.vasilakis@surrey.ac.uk
}

\begin{abstract}
In this paper, we propose a multirate teletraffic loss model of a single link with certain bandwidth capacity that accommodates Poisson arriving calls, which can tolerate bandwidth compression (elastic traffic), under the threshold policy. When compression occurs, the service time of new and in-service calls increases. The threshold policy provides different $\mathrm{QoS}$ among service-classes by limiting the number of calls of a service-class up to a predefined threshold, which can be different for each serviceclass. Due to the bandwidth compression mechanism, the steady state probabilities in the proposed model do not have a product form solution. However, we approximate the model by a reversible Markov chain, and prove recursive formulas for the calculation of call blocking probabilities and link utilization. The accuracy of the proposed formulas is verified through simulation and found to be very satisfactory.
\end{abstract}

Index Terms - Poisson Process, Elastic Calls, Threshold Policy, Call Blocking, Recursive Formula.

\section{INTRODUCTION}

$\mathrm{M}$ ultirate loss models based on recursive formulas provide an efficient way for the call-level QoS assessment in communication networks which accommodate elastic traffic. Elastic traffic refers to in-service calls whose bandwidth can be compressed, while their service time increases. The calllevel analysis of a link that behaves as a loss system and accommodates multirate elastic traffic is based on the classical Erlang Multirate Loss Model (EMLM) [1],[2].

In the EMLM, a link of capacity $C$ bandwidth units (b.u.) accommodates $K$ service-classes. Service-class $k$ calls $(k=1, \ldots, K)$ follow a Poisson process with arrival rate $\lambda_{k}$ and require a peak-bandwidth of $b_{k}$ b.u. Calls compete for the available link b.u. under the Complete Sharing (CS) policy. According to the CS policy, new calls are blocked only if their required b.u. are more than the available link b.u. Accepted calls remain in the link for an arbitrarily distributed service time [1]. The steady-state probabilities in the EMLM have a Product Form Solution (PFS). The latter leads to an accurate calculation of Call Blocking Probabilities (CBP) via the classical Kaufman-Roberts recursive formula [1],[2] which has led to numerous extensions of the EMLM (e.g., [3]-[19]).

Elastic traffic has been incorporated in the EMLM in [20].

We name the model of [20] Elastic EMLM (E-EMLM). In the E-EMLM, Poisson arriving calls of service-class $k$ have peak and minimum bandwidth requirements of $b_{k}$ and $b_{k, \text { min }}^{\prime}$ b.u., respectively. A new service-class $k$ call is accepted in the system with $b_{k}$ b.u. if the occupied link bandwidth, after the call's acceptance, does not exceed $C$. If $b_{k}$ is higher than the available bandwidth, then the system accepts this call by compressing its initial bandwidth $b_{k}$ together with the bandwidth of all in-service calls. Bandwidth compression of a service-class $k$ call is permitted down to $b_{k, \min }^{\prime}$. Call blocking occurs if $b_{k \text {,min }}^{\prime}$ is still higher than the available bandwidth. Bandwidth expansion occurs when a call, with compressed bandwidth, departs from the system. Then, the remaining calls expand their bandwidth in proportion to their peak-bandwidth.

In this paper, we propose the E-EMLM/TH by considering the E-EMLM and modifying the admission mechanism to include the threshold $(\mathrm{TH})$ policy [21]. In the TH policy, the number of in-service calls of service-class $k$ should not exceed a threshold, after the acceptance of a new service-class $k$ call. Otherwise, call blocking occurs. The importance of the $\mathrm{TH}$ policy in teletraffic engineering is twofold: i) It analyzes a multirate access tree network which accommodates calls of $K$ service-classes [21]. ii) It provides service-class differentiation in terms of CBP, revenue rates, etc. [22].

Applications of the TH policy are numerous (e.g., [23][27]). In [23], the TH policy is applied in a two-tier multirate wireless network. The optimal call admission policy in this network has a two dimensional threshold structure. In [24], [25], the TH policy provides QoS differentiation between new and handoff calls of the same service-class $k$ accommodated in the same cell. In [26], the TH policy provides service differentiation and achieves revenue optimization in a mobile cellular system. In [27], the TH policy is applied in the EMLM and a formula similar to the Kaufman-Roberts formula is proposed for the calculation of link occupancy distribution. We name the model of [27], EMLM/TH.

In the aforementioned papers, in-service calls cannot alter their bandwidth. To the best of our knowledge, this is the first paper that considers the TH policy in a system that services 
elastic calls. Bandwidth compression destroys reversibility in the proposed model and therefore no PFS exists. However, we resort to an approximate but reversible Markov chain and prove a recursive formula for the determination of the link occupancy distribution and, consequently, CBP and link utilization. The accuracy of the proposed formulas is verified through simulation and found to be very satisfactory.

The remainder of this paper is as follows: Section II, contains three subsections: In subsection II.A, we present the basic assumptions of the proposed model; the bandwidth compression mechanism is described via an example in subsection II.B, while in subsection II.C, we prove the recursive formula for the link occupancy distribution, provide formulas for the various performance measures and show the relationship of the proposed model with other loss models. In Section III, we provide numerical results whereby the new model is compared to the EMLM/TH and E-EMLM and evaluated through simulation. We conclude in Section IV.

\section{The Proposed Model (E-EMLM/TH)}

\section{A. The system model}

Consider a link of capacity $C$ b.u. that accommodates $K$ elastic service-classes. Service-class $k(k=1, \ldots, K)$ calls follow a Poisson process with arrival rate $\lambda_{k}$ and request $b_{k}$ b.u. Bandwidth compression is introduced in the system by allowing the occupied link bandwidth $j$ to virtually exceed $C$ up to $T$ b.u., i.e., $j=0,1, \ldots, T$. Let $\boldsymbol{n}=\left(n_{1}, \ldots, n_{K}\right)$ be the vector of all in-service calls and $\boldsymbol{b}=\left(b_{1}, \ldots, b_{K}\right)$ the vector of peak-bandwidth requirements, then $j=\boldsymbol{n} \boldsymbol{b}$.

The decision to accept a new service-class $k$ call in the system is based on the following constraints: a) The number of in-service calls of service-class $k, n_{k}$, together with the new call, should not exceed a threshold $n_{k}^{*}$, i.e., $n_{k}+1 \leq n_{k}^{*}$. Otherwise the call is blocked. This constraint expresses the TH policy. b) If constraint (a) is met then: b1) if $j+b_{k} \leq C$, the call is accepted in the system with $b_{k}$ b.u. and remains in the system for an exponentially distributed service time with mean $\mu_{k}^{-1}$. b2) if $T \geq j+b_{k}>C$ the call is accepted by compressing its $b_{k}$ together with the bandwidth of all inservice calls of all service-classes.

The compressed bandwidth of the new service-class $k$ call is:

$b_{k}^{\prime}=r b_{k}=C b_{k} /\left(j+b_{k}\right)$

where $r \equiv r(\boldsymbol{n})=C /\left(\boldsymbol{n} \boldsymbol{b}+b_{k}\right)=C /\left(j+b_{k}\right)$.

In order to keep constant the product service time by bandwidth per call, the mean value of the service time of the new serviceclass $k$ call changes to $1 / \mu_{k}^{\prime}=\left(j+b_{k}\right) / C \mu_{k}$.

The compressed bandwidth of all in-service calls becomes equal to $b_{i}^{\prime}=C b_{i} /\left(j+b_{k}\right)$ for $i=1, \ldots, K$. When all calls have compressed their bandwidth, then $j=C$. Note that the minimum bandwidth that a call of service-class $k$ tolerates is: $b_{k, \min }^{\prime}=r_{\min } b_{k}=C b_{k} / T$

where $r_{\min }=C / T$ is the minimum proportion of the required peak-bandwidth and is common for all service-classes.

A new service-class $k$ call, with $b_{k}$ b.u., is blocked if $j+b_{k}>T$.

When an in-service call, with compressed bandwidth $b_{i}^{\prime}$ departs from the system then the rest in-service calls expand their bandwidth to $b_{i}^{\prime \prime}$ in proportion to their $b_{i}$, as follows:

$b_{i}^{\prime \prime}=\min \left(b_{i}, b_{i}^{\prime}+b_{i} b_{k}^{\prime} / \sum_{k=1}^{K} n_{k} b_{k}\right)$

\section{B. A tutorial example}

The following example illustrates the compression/expansion mechanism. Let $C=4$ b.u., $T=8$. b.u., $K=2, \lambda_{1}=\lambda_{2}=1$ call/time unit, $b_{1}=2$ b.u., $b_{2}=4$ b.u and $\mu_{1}^{-1}=\mu_{2}^{-1}=1$ time unit. In-service calls of the $1^{\text {st }}$ service-class can be at most three, i.e., $n_{1}^{*}=3$. Similarly, let $n_{2}^{*}=1$ for the $2^{\text {nd }}$ service-class. This system has 7 states $\boldsymbol{n}=\left(n_{1}, n_{2}\right)$ presented in Fig. 1. Let us examine now the cases of a call arrival/departure.

* Call arrival. A new $2^{\text {nd }}$ service-class call arrives in the system while the state is $\left(n_{1}, n_{2}\right)=(2,0)$ and $j=4$ b.u. Since $j^{\prime}=j+b_{2}=T=8$ b.u., the call is accepted in the system after bandwidth compression has been applied to these three calls. In the new state, $\left(n_{1}, n_{2}\right)=(2,1)$, calls compress their bandwidth to: $b_{1, \min }^{\prime}=r(2,1) b_{1}=1.0, \quad b_{2, \text { min }}^{\prime}=r(2,1) b_{2}=2.0 \quad$ so that $j=C$. Similarly, the values of service time become $\mu_{1}^{-1} / r_{\min }=\mu_{2}^{-1} / r_{\min }=2.0$.

* Call departure. Let the system be in state $\left(n_{1}, n_{2}\right)=(2,1)$ when a $1^{\text {st }}$ service-class call departs from the system. Then, its bandwidth $b_{1, \min }^{\prime}=1.0$ is shared to the other two calls in proportion to their peak-bandwidth. So, in state $\left(n_{1}, n_{2}\right)=(1,1)$ we have: $b_{1}^{\prime}=4 b_{1} / 6=1.333$ b.u., $b_{2}^{\prime}=4 b_{2} / 6=2.667$ b.u.

Figure 1 shows the state transition diagram of this example. If we consider the states $\left(n_{1}, n_{2}\right):(1,0),(1,1),(2,1)$ and $(2,0)$ then the Kolmogorov's criterion (flow clockwise=flow counterclockwise) holds [21]. Thus, the Markov chain is irreversible and the E-EMLM/TH has no PFS. To circumvent this problem, we use state-dependent factors $\phi_{k}(\boldsymbol{n})$, which lead to a reversible Markov chain:

$\phi_{k}(\boldsymbol{n})=\left\{\begin{array}{l}1, \text { when } n b \leq C \text { and } \boldsymbol{n} \text { in } \boldsymbol{\Omega} \\ \frac{x\left(\boldsymbol{n}_{k}^{-}\right)}{x(\boldsymbol{n})}, \text { when } C<n b \leq T \text { and } \boldsymbol{n} \text { in } \boldsymbol{\Omega}\end{array}\right.$

where $\boldsymbol{\Omega}$ is the state space $\boldsymbol{\Omega}=\left\{\boldsymbol{n}: 0 \leq \boldsymbol{n} \boldsymbol{b} \leq T, n_{k} \leq n_{k}^{*}, k=1, \ldots, K\right\}$, $\boldsymbol{n}=\left(n_{1}, \ldots, n_{k}, \ldots, n_{K}\right), \boldsymbol{n}_{\boldsymbol{k}}^{-}=\left(n_{1}, \ldots, n_{k}-1, \ldots, n_{K}\right)$ and

$x(\boldsymbol{n})=\frac{1}{C} \sum_{k=1}^{K} n_{k} b_{k} x\left(\boldsymbol{n}_{k}^{-}\right)$, when $C<\boldsymbol{n} \boldsymbol{b} \leq T$, $\boldsymbol{n}$ in $\boldsymbol{\Omega}$ 


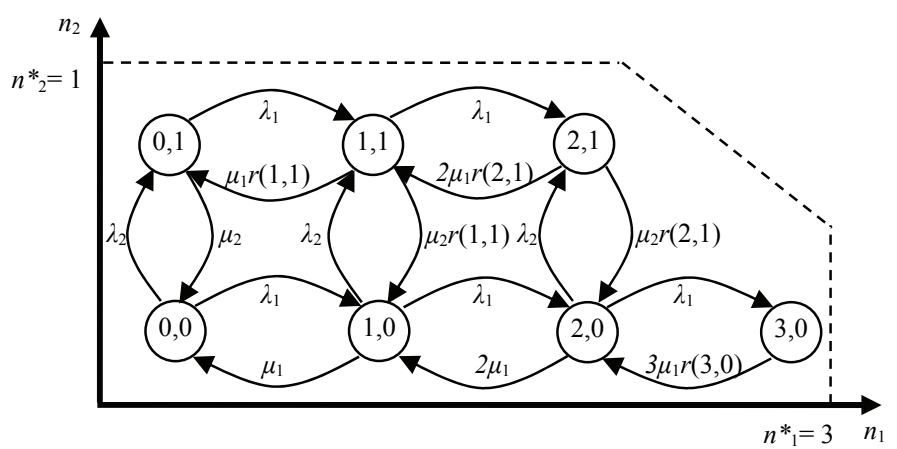

Figure 1. State transition diagram of the tutorial example.

Equation (5) ensures that $\sum_{k=1}^{K} n_{k} b_{k} \phi_{k}(\boldsymbol{n})=C$, when $T \geq \boldsymbol{n} \boldsymbol{b}>C$.

Figure 2 shows the modified state transition diagram, due to $\phi_{k}(\boldsymbol{n})$ 's, whereby the Kolmogorov's criterion holds.

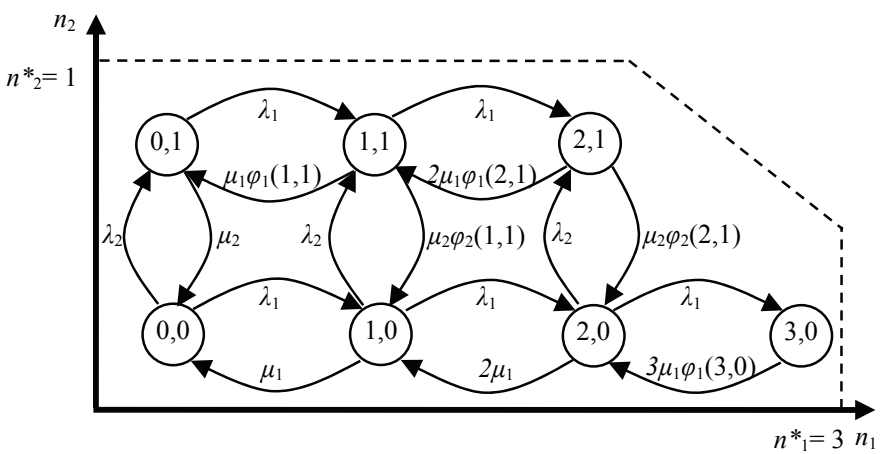

Figure 2. Modified state transition diagram of the tutorial example.

\section{The analytical model}

The following theorem provides a recursive formula for the calculation of the link occupancy distribution.

\section{Theorem}

The link occupancy distribution, $G(j)$, of the modified analytical model satisfies the following recursive formula:

$G(j)=\frac{1}{\min (C, j)} \sum_{k=1}^{K} a_{k} b_{k}\left[G\left(j-b_{k}\right)-T_{k}\left(j-b_{k}\right)\right]$, for $j=1, \ldots, T$

where: $G(0)=1, G(y)=0$ for $y<0$.

Proof

The global balance equation for state $\boldsymbol{n}$, expressed as rate into state $\boldsymbol{n}=$ rate out of state $\boldsymbol{n}$, is given by:

$\sum_{k=1}^{K} \lambda_{k} P\left(\boldsymbol{n}_{k}^{-}\right)+\sum_{k=1}^{K}\left(n_{k}+1\right) \mu_{k} \phi_{k}\left(\boldsymbol{n}_{k}^{+}\right) P\left(\boldsymbol{n}_{k}^{+}\right)=\sum_{k=1}^{K} \lambda_{k} P(\boldsymbol{n})+\sum_{k=1}^{K} n_{k} \mu_{k} \phi_{k}(\boldsymbol{n}) P(\boldsymbol{n})$ where: $\boldsymbol{n}_{k}^{+}=\left(n_{1}, \ldots, n_{k}+1, \ldots, n_{K}\right)$ and $P(\boldsymbol{n}), P\left(\boldsymbol{n}_{k}^{-}\right), P\left(\boldsymbol{n}_{k}^{+}\right)$are the probability distributions of states $\boldsymbol{n}, \boldsymbol{n}_{k}^{-}, \boldsymbol{n}_{k}^{+}$, respectively.

Assume now, the existence of Local Balance (LB) between adjacent states. Then the following LB equations (rate up=rate down) can be extracted, for $k=1, \ldots, K$ and $\boldsymbol{n} \in \boldsymbol{\Omega}$ :

$\lambda_{k} P\left(\boldsymbol{n}_{k}^{-}\right)=n_{k} \mu_{k} \phi_{k}(\boldsymbol{n}) P(\boldsymbol{n})$
$\lambda_{k} P(\boldsymbol{n})=\left(n_{k}+1\right) \mu_{k} \phi_{k}\left(\boldsymbol{n}_{k}^{+}\right) P\left(\boldsymbol{n}_{k}^{+}\right)$
Based on the assumption of LB, $P(\boldsymbol{n})$ has the solution:

$P(\boldsymbol{n})=G^{-1}\left(x(\boldsymbol{n}) \prod_{k=1}^{K} \frac{a_{k}^{n_{k}}}{n_{k} !}\right)$

where $a_{k}=\lambda_{k} / \mu_{k}$ is the offered traffic-load (in erl) of service-

class $k$ and $G \equiv G(\boldsymbol{\Omega})=\sum_{\boldsymbol{n} \in \boldsymbol{\Omega}}\left(x(\boldsymbol{n}) \prod_{k=1}^{K} \frac{a_{k}^{n_{k}}}{n_{k} !}\right)$.

Since $j$ is the occupied link bandwidth, $G(j)$ is defined as:

$G(j)=\sum_{\boldsymbol{n} \in \boldsymbol{\Omega}_{j}} P(\boldsymbol{n}), \quad \boldsymbol{\Omega}_{j}=\{\boldsymbol{n} \in \boldsymbol{\Omega}: \boldsymbol{n} \boldsymbol{b}=j\}$

Consider now two sets: (1) $0 \leq j \leq C$ and (2) $C<j \leq T$. For set (1), we have the EMLM/TH and $G(j)$ 's are given by the following accurate and recursive formula [27]:

$G(j)=\frac{1}{j} \sum_{k=1}^{K} a_{k} b_{k}\left[G\left(j-b_{k}\right)-T_{k}\left(j-b_{k}\right)\right]$, for $j=1, \ldots, C$

where:

$T_{k}(x):=\operatorname{Pr}\left[j=x, n_{k}=n_{k}^{*}\right]$

In (12) the fact that $n_{k}=n_{k}^{*}$ implies that $j \geq n_{k}^{*} b_{k}$.

When $C<j \leq T$, we substitute (4) in (7) to have:

$a_{k} x(\boldsymbol{n}) P\left(\boldsymbol{n}_{k}^{-}\right)=n_{k} x\left(\boldsymbol{n}_{k}^{-}\right) P(\boldsymbol{n})$

Multiplying both sides of (13) by $b_{k}$ and summing over $k$ we obtain:

$x(\boldsymbol{n}) \sum_{k=1}^{K} a_{k} b_{k} P\left(\boldsymbol{n}_{k}^{-}\right)=P(\boldsymbol{n}) \sum_{k=1}^{K} n_{k} b_{k} x\left(\boldsymbol{n}_{k}^{-}\right)$

Equation (14), due to (6) is written as:

$P(\boldsymbol{n})=\frac{1}{C} \sum_{k=1}^{K} a_{k} b_{k} P\left(\boldsymbol{n}_{k}^{-}\right)$

Summing both sides of (15) over $\boldsymbol{\Omega}_{j}=\{\boldsymbol{n} \in \boldsymbol{\Omega}: \boldsymbol{n} \boldsymbol{b}=j\}$ and based on (10), we obtain:

$G(j)=\frac{1}{C} \sum_{k=1}^{K} a_{k} b_{k} \sum_{\boldsymbol{n} \in \boldsymbol{\Omega}_{j}} P\left(\boldsymbol{n}_{k}^{-}\right)$

Since $n_{k} \leq n_{k}^{*}$ then $\sum_{\boldsymbol{n} \in \boldsymbol{\Omega}_{j}} P\left(\boldsymbol{n}_{k}^{-}\right)=G\left(j-b_{k}\right)-\operatorname{Pr}\left[x=j-b_{k}, n_{k}=n_{k}^{*}\right]$.

Thus, (16) can be written as:

$G(j)=\frac{1}{C} \sum_{k=1}^{K} a_{k} b_{k}\left[G\left(j-b_{k}\right)-T_{k}\left(j-b_{k}\right)\right]$

where $T_{k}(x)$ is given by (12).

Equations (11), (17) give (6) (End of Proof)

Having determined $G(j)$ 's we can calculate the CBP of service-class $k, B_{k}$, and the link utilization, $U$, as follows:

$B_{k}=\sum_{j=T-b_{k}+1}^{T} G^{-1} G(j)+\sum_{j=n_{k} b_{k}}^{T-b_{k}} G^{-1} T_{k}(j)$
$U=\sum_{j=1}^{C} j G^{-1} G(j)+\sum_{j=C+1}^{T} C G^{-1} G(j)$ 
where $G=\sum_{j=0}^{T} G(j)$ is the normalization constant.

In (6) and (18) the knowledge of $T_{k}(j)$ is required. Since $T_{k}(j)>0$ when $j=n_{k}^{*} b_{k}, \ldots, T-b_{k}$, we consider two subsets: 1$)$ $n_{k}^{*} b_{k} \leq j \leq C$ and 2) $C+1 \leq j \leq T-b_{k}$.

For the first subset, let a system of capacity $F_{k}=T-b_{k}-n_{k}^{*} b_{k}$ that accommodates all service-classes but service-class $k$. For this system, we define $r_{k}(j)$ as follows:

$r_{k}(j)=\frac{1}{j} \sum_{\substack{i=1 \\ i \neq k}}^{K} a_{i} b_{i}\left[r_{k}\left(j-b_{i}\right)-T_{i}\left(j-b_{i}\right)\right]$, for $j=1, \ldots, F_{k}$

Based on $r_{k}(j)$ 's, we compute $T_{k}(j)$ via the formula:

$T_{k}(j)=\frac{a_{k}^{n_{k}^{*}}}{n_{k}^{*} !} r_{k}\left(j-n_{k}^{*} b_{k}\right)$

For the second subset, $T_{k}(j)$ can be determined by:

$T_{k}(j)=\frac{a_{k}^{n_{k}^{*}}}{n_{k}^{*} !} \sum_{n \in \Omega} x(\boldsymbol{n}) \prod_{\substack{i=1 \\ i \neq k}}^{K} \frac{a_{i}^{n_{i}}}{n_{i} !}$

where: $\boldsymbol{\Omega}^{\prime}=\left\{\boldsymbol{n} \in \boldsymbol{\Omega}^{\prime}: n_{k}^{*} b_{k}+\sum_{i=1, i \neq k}^{K} n_{i} b_{i}=j, C+1 \leq j \leq T-b_{k}\right\}$.

To show the relationship of the proposed model with other multirate loss models, we distinguish three cases:

a) If we do not consider the TH policy in the E-EMLM/TH then $G(j)$ 's are given by the E-EMLM, [20]:

$G(j)=\frac{1}{\min (C, j)} \sum_{k=1}^{K} a_{k} b_{k} G\left(j-b_{k}\right)$, for $j=1, \ldots, T$

The CBP of service-class $k, B_{k}$, is given by:

$$
B_{k}=\sum_{j=T-b_{k}+1}^{T} G^{-1} G(j)
$$

The link utilization can be determined by (19).

b) If $C=T$ and the TH policy is considered, then the EMLM/TH occurs [27]. The CBP of service-class $k, B_{k}$, and the link utilization $U$ are given by:

$$
\begin{aligned}
& B_{k}=\sum_{j=C-b_{k}+1}^{C} G^{-1} G(j)+\sum_{j=n_{k}^{*} b_{k}}^{C-b_{k}} G^{-1} T_{k}(j) \\
& U=\sum_{j=1}^{C} j G^{-1} G(j)
\end{aligned}
$$

c) If $C=T$ and we do not consider the TH policy, then the EEMLM/TH coincides with the EMLM. In that case, $G(j)$ 's are given by the Kaufman-Roberts recursion [1], [2]:

$$
G(j)=\frac{1}{j} \sum_{k=1}^{K} a_{k} b_{k} G\left(j-b_{k}\right), \text { for } j=1, \ldots, C
$$

The CBP of service-class $k$ is given by:

$$
B_{k}=\sum_{j=C-b_{k}+1}^{C} G^{-1} G(j)
$$

while the link utilization can be determined by (26).

\section{NUMERICAL RESUlTS - EVALUATION}

In this section, we present an application example of the proposed model (E-EMLM/TH) and the models of [20], [27] (E-EMLM and EMLM/TH, respectively). Through the proposed model we obtain analytical CBP and link utilization results, and compare them with the corresponding simulation results, in order to reveal the accuracy of the E-EMLM/TH. The simulation model is based on the bandwidth compression mechanism described by $r(\boldsymbol{n})$ 's. Simulation results, based on SIMSRIPT III [28], are mean values of 7 runs. Each run is based on the generation of five million calls. To account for a warm-up period, the blocking events of the first $5 \%$ of these calls are not considered in the results. Since reliability ranges are very small, they are not presented in the figures that follow.

Consider a link of capacity $C=70$ b.u. and three values of $T$ : 1) $T=C=70$ b.u., 2) $T=75$ b.u. and 3) $T=80$ b.u. The link accommodates the following three service-classes:

$1^{\text {st }}$ service-class: $a_{1}=5$ erl, $b_{1}=2$ b.u., $n_{1}^{*}=n_{1, \max }=25$

$2^{\text {nd }}$ service-class: $a_{2}=1.5$ erl, $b_{2}=5$ b.u., $n_{2}^{*}=n_{2, \max }=11$

$3^{\text {rd }}$ service-class: $a_{3}=1.0$ erl, $b_{3}=9$ b.u., $n_{3}^{*}=n_{3, \max }=6$

In the x-axis of Figs. 5-11, traffic loads $a_{1}, a_{2}$ and $a_{3}$ increase in steps of 1.0, 0.5 and $0.25 \mathrm{erl}$, respectively. In this way, Point 1 represents the vector $\left(a_{1}, a_{2}, a_{3}\right)=(5.0,1.5,1.0)$ while Point 7 is $\left(a_{1}, a_{2}, a_{3}\right)=(11.0,4.5,2.5)$.

In Figs. 5-7, we consider the proposed E-EMLM/TH and present the analytical and simulation $\mathrm{CBP}$ results of all service-classes, for all values of $T$. For comparison, we present the corresponding analytical results of the EMLM/TH. Based on Figs. 5-7, we see that: i) the results obtained by the proposed formulas are close to the simulation results. ii) The bandwidth compression mechanism reduces CBP of all service-classes. iii) The analytical results of the EMLM/TH fail to approximate the simulation results of the E-EMLM/TH.

In Fig. 8, we present the corresponding link utilization results (in b.u.). The link utilization is higher when $T=80$ b.u., a result that is expected since this value of $T$ achieves the highest CBP reduction (compared to $T=70$ or 75 b.u.).

In Figs. 9-11, we consider the E-EMLM/TH together with the E-EMLM and present the analytical CBP results of all service-classes for $T=75$ b.u. and $n_{3, \max }=3,4$ and 5 calls. The existing E-EMLM fails to approximate the CBP results obtained by the E-EMLM/TH, in the cases of $n_{3, \max }=3,4$.

The fact that the two models give quite close CBP results for $n_{3, \max }=5$ is explained as follows: Assuming that only calls of the $3^{\text {rd }}$ service-class exist in the link then the theoretical max. number of the $3^{\text {rd }}$ service-class calls is 8 (each of which occupies $(70 / 75) * 9=8.4$ b.u.). Approaching this value makes the E-EMLM/TH behave as the E-EMLM. We also see that the increase of $n_{3, \max }$ results in the CBP increase for the $1^{\text {st }}$ and $2^{\text {nd }}$ service-classes (Fig. 9 and Fig. 10, respectively) and the decrease of CBP for the $3^{\text {rd }}$ service-class (Fig. 11). 


\section{CONCLUSION}

In this paper we propose a multirate loss model where Poisson arriving calls of different elastic service-classes compete for the available link bandwidth under the threshold policy. Calls can tolerate bandwidth compression and expansion. The analysis of the proposed model leads to approximate but recursive formulas for the calculation of $\mathrm{CBP}$ and link utilization. Simulation results verify the accuracy of the proposed model. In addition, numerical results show the necessity of the proposed model, since existing models fail to approximate the results obtained by the proposed model.

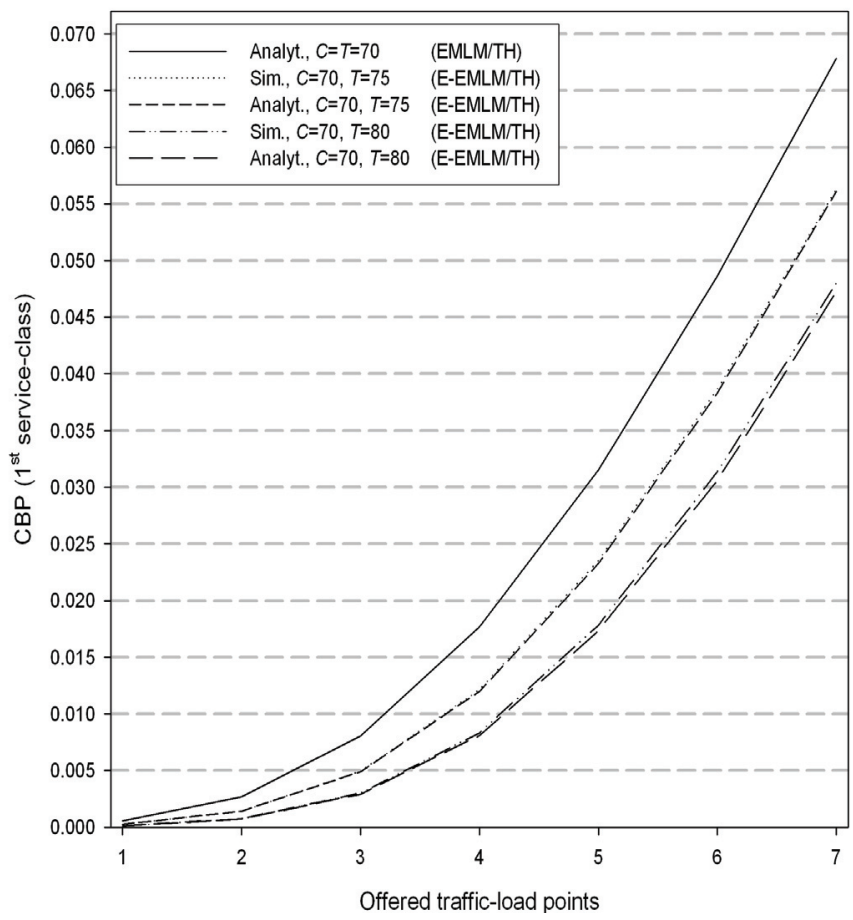

Figure $5 . \mathrm{CBP}$ of the $1^{\text {st }}$ service-class, when $n_{3, \max }=6$.

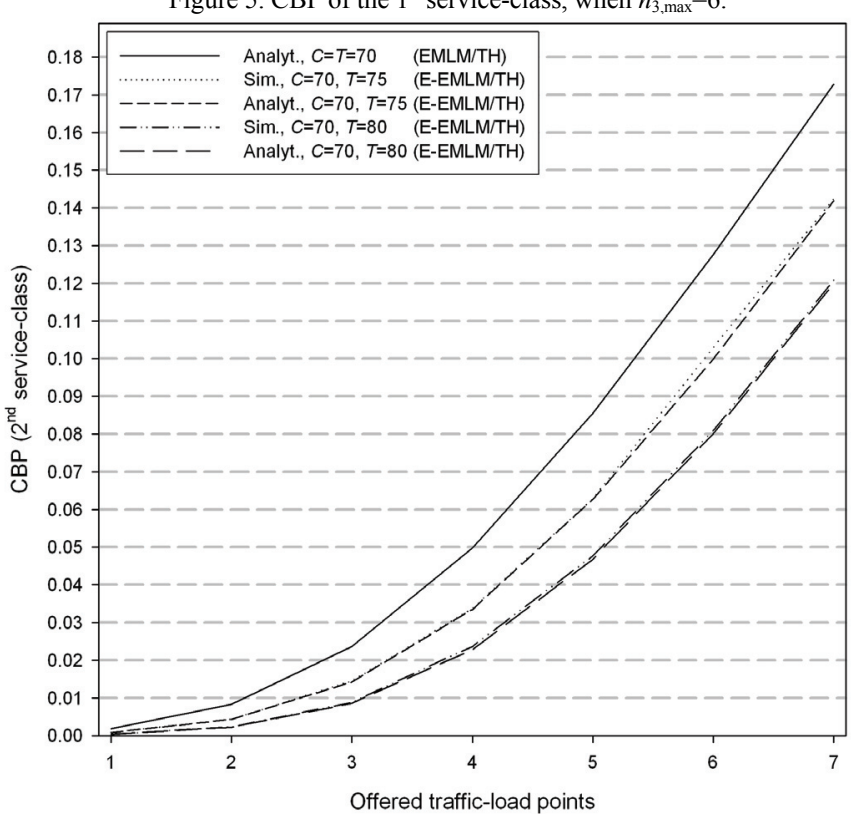

Figure 6. CBP of the $2^{\text {nd }}$ service-class, when $n_{3, \max }=6$.

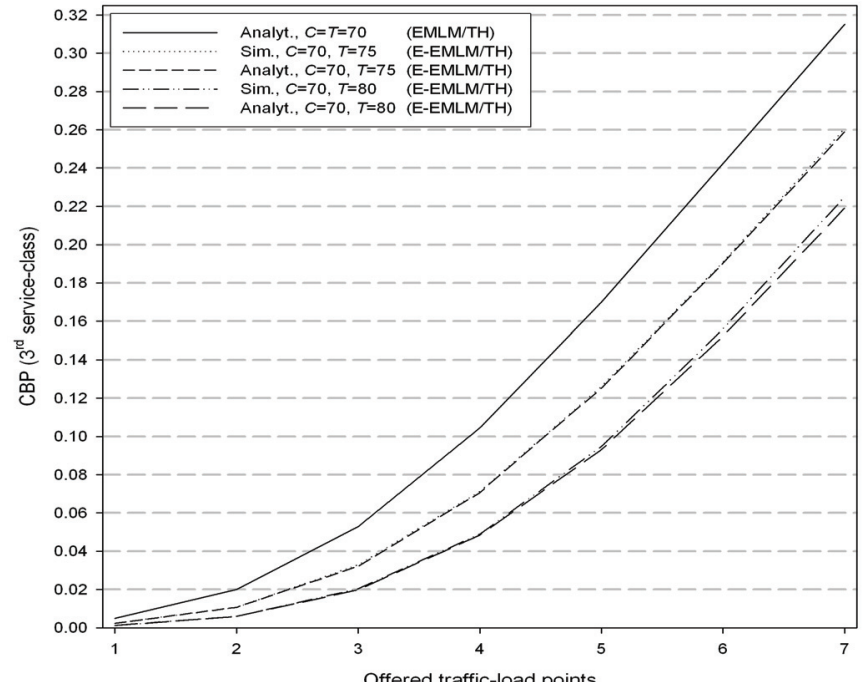

Figure 7. CBP of the $3^{\text {rd }}$ service-class, when $n_{3, \max }=6$.

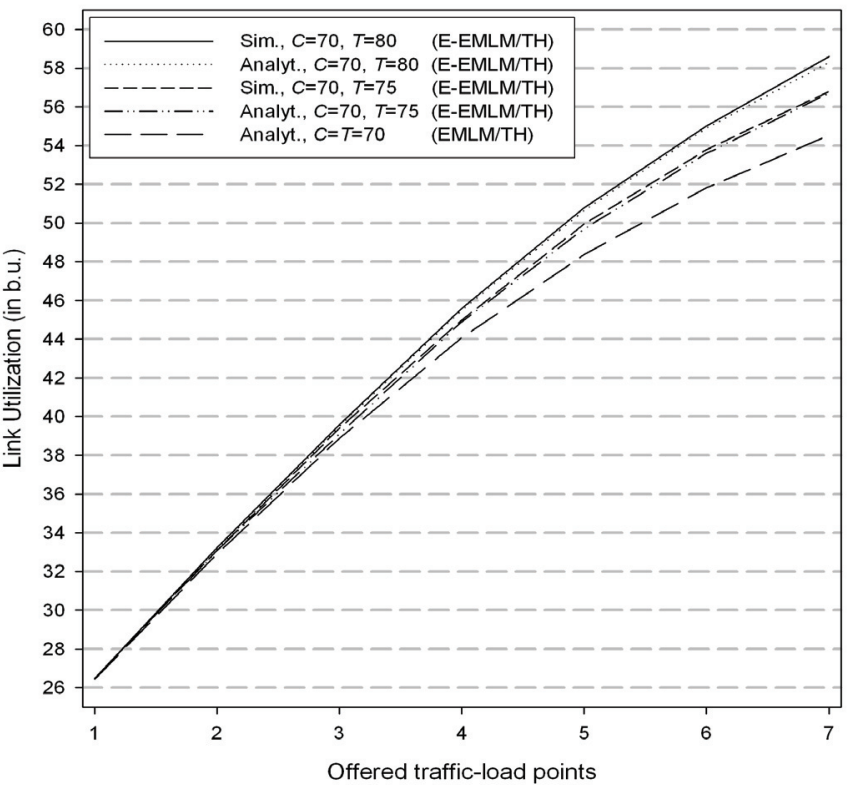

Figure 8: Link Utilization, when $n_{3, \max }=6$.

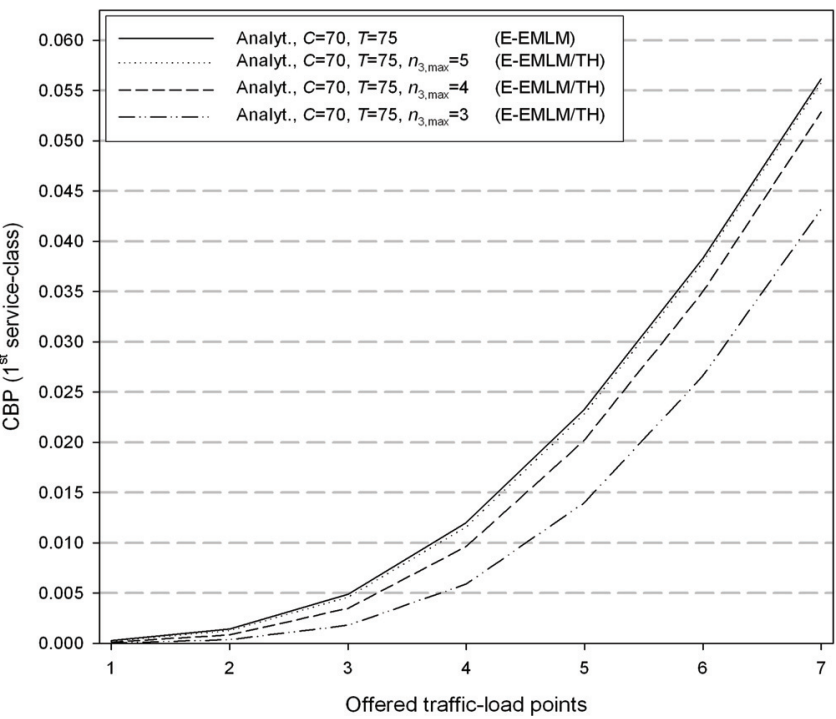

Figure 9 . CBP of the $1^{\text {st }}$ service-class, when $n_{3, \max }=3,4$ and 5 . 


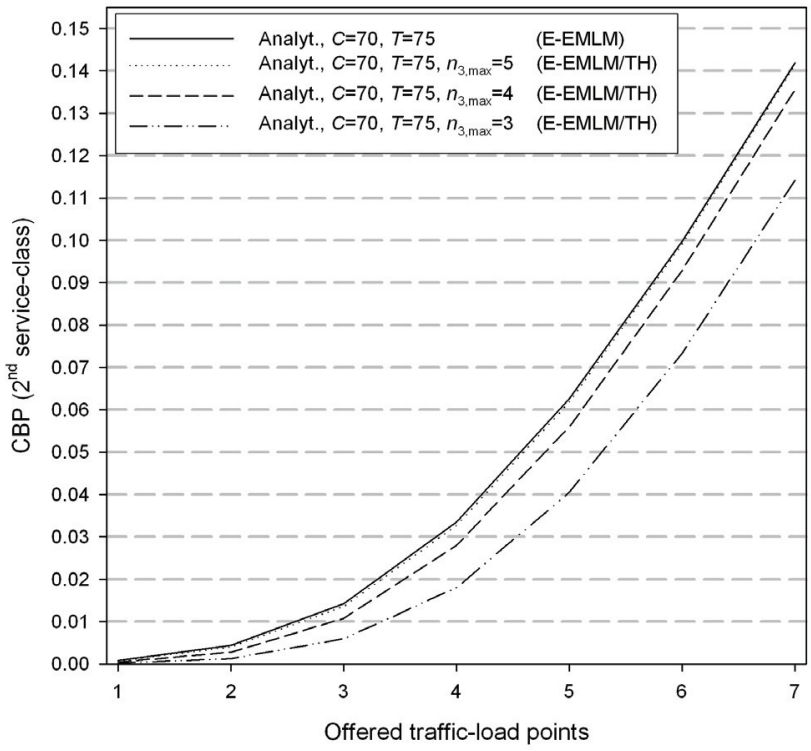

Figure 10 . CBP of the $2^{\text {nd }}$ service-class, when $n_{3, \max }=3,4$ and 5 .

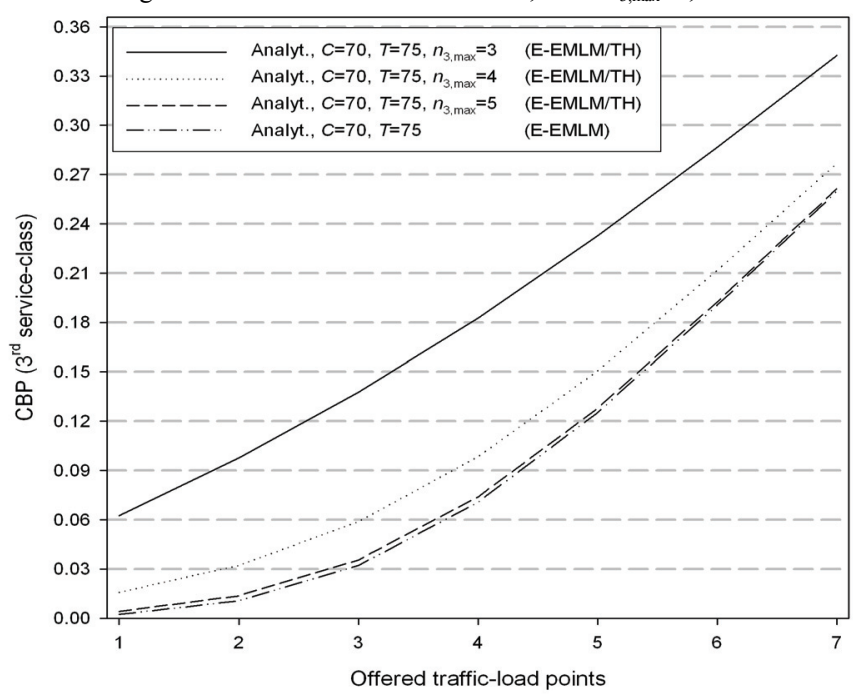

Figure 11. CBP of the $3^{\text {rd }}$ service-class, when $n_{3, \max }=3,4$ and 5 .

\section{REFERENCES}

[1] J. Kaufman, "Blocking in a shared resource environment", IEEE Trans. Commun., vol. 29, no. 10, pp. 1474-1481, October 1981.

[2] J. W. Roberts, "Teletraffic models for the Telecom 1 integrated services network", Proc. ITC-10, Mondreal, Canada, 1982

[3] J. Kaufman, "Blocking with retrials in a completely shared resource environment", Perf. Eval., vol. 15, no. 2, pp. 99-113, June 1992.

[4] I. Moscholios, M. Logothetis and G. Kokkinakis, "Connection dependent threshold model: a generalization of the Erlang multiple rate loss model", Performance Evaluation, vol.48, issues 1-4, pp. 177-200, May 2002.

[5] I. Moscholios, M. Logothetis and M. Koukias, "An ON-OFF multi-rate loss model of finite sources”, IEICE Trans. Commun., vol. E90-B, no. 7 , pp.1608-1619, July 2007.

[6] M. Glabowski, A. Kaliszan and M. Stasiak, "Modeling product-form state depedent systems with BPP traffic", Performance Evaluation, vol. 67, issue 3, pp. 174-197, March 2010.

[7] I. Moscholios, J. Vardakas, M. Logothetis and A. Boucouvalas, "A batched Poisson multirate loss model supporting elastic traffic under the bandwidth reservation policy", Proc. IEEE ICC, Kyoto, Japan, June 2011.
[8] I. Moscholios, J. Vardakas, M. Logothetis and A. Boucouvalas, "QoS guarantee in a batched Poisson multirate loss model supporting elastic and adaptive traffic", Proc. IEEE ICC, Ottawa, Canada, June 2012.

[9] M. Glabowski and M. D. Stasiak, "Internal blocking probability calculation in switching networks with additional inter-stage links and mixture of Erlang and Engset traffic", Image Processing \& Communication, vol. 17, no. 1-2, pp. 67-80, January 2013.

[10] I. Moscholios, J. Vardakas, M. Logothetis and A. Boucouvalas, "Congestion probabilities in a batched Poisson multirate loss model supporting elastic and adaptive traffic", Annals of Telecommunications, vol. 68, issue 5, pp. 327-344, June 2013.

[11] S. Hanczewski, M. Stasiak and J.Weissenberg, "A queueing model of a multi-service system with state-dependent distribution of resources for each class of calls", IEICE Trans. Commun., vol. E97-B, no. 8, pp.15921605, August 2014

[12] F. Cruz-Pérez, J. Vázquez-Ávila and L. Ortigoza-Guerrero, "Recurrent formulas for the multiple fractional channel reservation strategy in multiservice mobile cellular networks", IEEE Communications Letters, vol. 8, no. 10, pp. 629-631, October 2004.

[13] V. Vassilakis, G. Kallos, I. Moscholios and M. Logothetis, "Call-level analysis of W-CDMA networks supporting elastic services of finite population”, Proc. IEEE ICC, Beijing, China, May 2008.

[14] M. Glabowski, M. Stasiak, A. Wisniewski, and P. Zwierzykowski, "Blocking probability calculation for cellular systems with WCDMA radio interface servicing $\mathrm{PCT} 1$ and PCT2 multirate traffic", IEICE Trans. Commun., vol.E92-B, pp.1156-1165, April 2009.

[15] I. Moscholios, G. Kallos, V. Vassilakis and M. Logothetis, "Congestion probabilities in CDMA-based networks supporting batched Poisson input traffic", Wireless Personal Communications, vol. 79, issue 2, pp. 1163-1186, November 2014.

[16] J. Vardakas, I. Moscholios, M. Logothetis and V. Stylianakis, "An analytical approach for dynamic wavelength allocation in WDM-TDMA PONs Servicing ON-OFF Traffic", IEEE/OSA Journal of Optical Commun. Networking, vol. 3, no. 4, pp. 347-358, April 2011.

[17] N. Jara and A. Beghelli, "Blocking probability evaluation of end-to-end dynamic WDM networks", Photonic Network Communications, vol. 24, issue 1, pp. 29-38, August 2012.

[18] J. Vardakas, I. Moscholios, M. Logothetis and V. Stylianakis, "Blocking performance of multi-rate OCDMA PONs with QoS guarantee", Int. Journal on Advances in Telecommunications, vol. 5, no. 3\&4, pp. 120130, December 2012.

[19] J. Vardakas, I. Moscholios, M. Logothetis, and V. Stylianakis, "Performance analysis of OCDMA PONs supporting multi-rate bursty traffic", IEEE Trans. on Commun., vol. 61, issue 8, pp. 3374-3384, August 2013

[20] G. Stamatelos and V. Koukoulidis, "Reservation - based bandwidth allocation in a radio ATM Network", IEEE/ACM Trans. Networking, vol. 5, pp.420-428, June 1997.

[21] K. Ross, Multiservice loss models for broadband telecommunication networks, Springer, 1995.

[22] J. Ni, D. Tsang, S. Tatikonda and B. Bensaou, "Optimal and structured call admission control policies for resource-sharing systems", IEEE Trans. on Commun., vol. 55, no. 1, January 2007.

[23] A. Farbod and B. Liang, "Efficient structured policies for admission control in heterogeneous wireless networks", ACM/Springer Mobile Networks and Applications (MONET), vol. 12, no. 5, pp. 309-323, 2007.

[24] T. Kwon, S. Kim, Y. Choi, M. Naghshineh, "Threshold-type call admission control in wireless/mobile multimedia networks using prioritised adaptive framework", IEE Electronics Letters, vol. 36, issue 9, pp. 852-854, Apr. 2000.

[25] N. Nasser, H. Hassanein, "Connection-level performance analysis for adaptive bandwidth allocation in multimedia wireless cellular networks", Proc. IEEE Int. Conf. on Performance, Computing and Communications, pp. 61-68, April 2004.

[26] J. Ni and S. Tatikonda, "Revenue optimization via call admission control and pricing for mobile cellular systems", Proc. IEEE ICC, vol. 5, pp. 3359-3364, May 2005

[27] D. Tsang and K. Ross, "Algorithms to determine exact blocking probabilities for multirate tree networks", IEEE Trans. Commun., vol. 38, issue 8, pp. 1266-1271, August 1990.

[28] Simscript II.5, http://www.simscript.com/ 